\title{
Clinical Manifestation and Laboratory Characteristics of SARS-CoV-2 Infection in Pregnant Women
}

\author{
Chunchen $\mathrm{Wu}^{1} \cdot$ Wenzhong Yang ${ }^{2} \cdot{\text { Xiaoxue } \mathrm{Wu}^{1} \cdot \text { Tianzhu Zhang }}^{1} \cdot$ Yaoyao Zhao ${ }^{1} \cdot$ Wei Ren $^{3}$ (1) \\ Jianbo Xia ${ }^{1}$ (1)
}

Received: 6 March 2020 / Accepted: 10 April 2020 / Published online: 20 April 2020

(c) Wuhan Institute of Virology, CAS 2020

\begin{abstract}
The severe acute respiratory syndrome coronavirus 2 (SARS-CoV-2) epidemic has become a major challenge to public health in China and other countries, considering its pathogenicity across all age groups. Pregnancy is a unique physiological condition, and is characterized by altered immunity and elevated hormone levels to actively tolerate the semiallogeneic fetus, which undergoes a sudden and substantial fluctuation during the immediate postpartum period. Changes in clinical features, laboratory characteristics, and imaging features of pregnant women during the pre-partum and postpartum periods require further elucidation. Here, we retrospectively analyzed the clinical features, laboratory characteristics, and imaging features of eight pregnant cases of SARS-CoV-2 infection during the pre-partum and post-partum periods. Our results showed that four of the eight pregnant women were asymptomatic before delivery but became symptomatic post-partum. Correspondingly, white blood cell (WBC) counts increased and lymphocyte (LYMPH) counts decreased. C-reactive protein (CRP) levels in the serum also increased to a higher level than those in general pregnancy. Therefore, it is imperative to closely monitor laboratory parameters including the WBC count, LYMPH count, and CRP, along with other imaging features in chest $\mathrm{CT}$ scans, to promptly prevent, diagnose, and treat a SARS-CoV-2 infection during pregnancy.
\end{abstract}

Keywords SARS-CoV-2 $\cdot$ Pregnant women $\cdot$ Clinical manifestation $\cdot$ Laboratory characteristics $\cdot$ Immunity

\section{Introduction}

Since the first report of pneumonia associated with the severe acute respiratory syndrome coronavirus 2 (SARSCoV-2) in Wuhan, Hubei Province, China in December 2019, the SARS-CoV-2 epidemic has become a major

Electronic supplementary material The online version of this article (https://doi.org/10.1007/s12250-020-00227-0) contains supplementary material, which is available to authorized users.

Wei Ren

ren33107@163.com

$\triangle$ Jianbo Xia

xjb915@126.com

1 Department of Laboratory Medicine, Maternal and Child Health Hospital of Hubei Province, Wuhan 430070, China

2 Department of Radiology, Maternal and Child Health Hospital of Hubei Province, Wuhan 430070, China

3 Department of Obstetrics, Maternal and Child Health Hospital of Hubei Province, Wuhan 430070, China public health challenge in China and other countries (Huang et al. 2020; Hui et al. 2020; Lu et al. 2020).

People of all ages are susceptible to SARS-CoV-2; however, current data have confirmed that adults, specifically older men with comorbidities, are more likely to be affected, suffering subsequently from severe pneumonia, pulmonary edema, acute respiratory distress syndrome, multiple organ failure, and death (Chen N et al. 2020; Huang et al. 2020; Wang et al. 2020). Pregnancy is a special physiological condition with unique characteristics, including altered immunity and hormone levels which is required to tolerate and support the development and survival of the placenta and fetus in the hostile maternal immune system environment (Robinson and Klein 2012). Compared with non-pregnant women, pregnant women are more severely affected by pathogenic infections, particularly those caused by respiratory pathogens, including the influenza and SARS viruses (Kourtis et al. 2014; Sappenfield et al. 2013; Wong et al. 2004). A study had previously reported SARS-CoV-2 infection in nine pregnant women 
(Chen $\mathrm{H}$ et al. 2020). However, the study primarily focused on the clinical characteristics and vertical transmission potential of SARS-CoV-2 infection in pregnant women. The changes in clinical features, laboratory characteristics, and imaging features of the pregnant women both before and after the delivery were not explored.

We retrospectively collected and analyzed detailed clinical data from eight pregnant patients, including six laboratory-confirmed SARS-CoV-2 infections and two highly suspected SARS-CoV-2 infection cases at the Maternal and Child Health Hospital of Hubei Province, Wuhan, China. The differences in clinical features, laboratory characteristics, and imaging features of pregnant women at pre-partum and post-partum were determined by examining the clinical features, laboratory characteristics, and imaging features of these patients.

\section{Methods}

\section{Study Design and Patients}

We conducted a retrospective review of medical records of eight pregnant patients, including six laboratory-confirmed SARS-CoV-2 infections and two highly suspected SARSCoV-2 infection cases, and five pregnant cases excluding SARS-CoV-2 infection admitted to the Maternal and Child Health Hospital of Hubei Province, Wuhan, China, from January 23 to February 10, 2020. The diagnosis was based on the World Health Organization interim guidance, and the New Coronavirus Pneumonia Prevention and Control Program published by the National Health Commission of China (National Health Commission of the PRC 2020; WHO 2020).

\section{Data Collection}

The clinical records, laboratory findings, chest CT scans, and treatment and outcome data for all patients were reviewed and collected with data collection forms. Two study investigators independently reviewed the data collection forms to verify the data accuracy. The date of disease onset was defined as the day when the symptoms were identified.

Throat swab samples were collected and dispatched to the Hubei Provincial Center for Disease Control and Prevention for testing for SARS-CoV-2. A positive test result indicated a confirmed diagnosis of SARS-CoV-2 infection.

\section{Results}

The eight pregnant women were in their third trimester, six underwent cesarean section, and two underwent eutocia (Table 1). Their ages ranged from 26 to 35 years, and the range of gestational weeks at admission was from 33 weeks ( +6 days) to 40 weeks ( +4 days).

Seven of the eight patients had no fever, cough, or other symptoms before delivery. Three of the seven patients developed fever at the 1 or 2 days postpartum, and one demonstrated the typical ground-glass opacities in the lungs on chest CT scans as described previously (Huang et al. 2020) (Table 1; Fig. 1). The remaining four patients did not present with symptoms after delivery. Among which, three had typical ground-glass opacities in the lungs in chest CT scans before delivery and one developed ground-glass opacities in chest CT scans after delivery (Table 1; Fig. 1). One of the eight patients had fever and typical ground-glass opacities on admission at 1-day prepartum. None of the patients had a high fever (body temperature $>39{ }^{\circ} \mathrm{C}$ ) or developed a severe pneumonia, requiring intensive unit care or mechanical ventilation (Table 1).

Laboratory test outcomes suggested that the white blood cell (WBC) counts of all eight patients were normal (patients 1-4 and 6-8) or slightly higher than the upper normal limit (patient 5) before delivery (Fig. 2A). However, the WBC counts of six patients drastically increased (patients $1-3,5-6$, and 8 ) after delivery, specifically for patients $1-2$ and 5-6 (Fig. 2A). In contrast, a slight increase in WBC counts was observed in pregnant patients, excluding those with SARS-CoV-2 infection after delivery (Fig. 2B). The lymphocyte (LYMPH) counts were normal (patients 1-6 and 8) or slightly lower than the normal lower limit (patient 7) before delivery (Fig. 2A). However, the LYMPH counts of five patients drastically decreased (patients 1-3, 5-6) lower than the normal lower limit after delivery (Fig. 2A). In contrast, there were no obvious changes in the LYMPH counts of the pregnant patients without the SARS-CoV-2 infection between pre-partum and post-partum (Fig. 2B).

Furthermore, we closely monitored the C-reactive protein (CRP) levels, since it is an important marker and gauge of inflammation in the body (Wu et al. 2015). Its levels were normal and comparable between pregnant women with SARS-CoV-2 infection and pregnant women without the SARS-CoV-2 infection before delivery (Fig. 3A). Patient CRP levels drastically increased postpartum. However, the mean serum CRP level was higher in patients with SARS-CoV-2 infection than in those without (Fig. 3A and Supplementary Table S1). D-dimer is one of the most important procoagulant markers, which may be elevated during viral infections (Subramaniam and Scharrer 2018). 
Table 1 Baseline characteristics of 8 pregnant women infected with severe acute respiratory syndrome coronavirus 2 (SARS-CoV-2).

\begin{tabular}{|c|c|c|c|c|c|c|c|c|}
\hline Characteristic & Patient 1 & Patient 2 & Patient 3 & Patient 4 & Patient 5 & Patient 6 & Patient 7 & Patient 8 \\
\hline Age (years) & 28 & 31 & 30 & 30 & 30 & 26 & 29 & 35 \\
\hline Gestational age on admission & 39 weeks & $\begin{array}{l}38 \text { weeks, } \\
1 \text { day }\end{array}$ & $\begin{array}{l}39 \text { weeks, } \\
1 \text { day }\end{array}$ & $\begin{array}{l}36 \text { weeks, } \\
4 \text { days }\end{array}$ & $\begin{array}{l}37 \text { weeks, } \\
6 \text { days }\end{array}$ & $\begin{array}{l}40 \text { weeks, } \\
3 \text { days }\end{array}$ & $\begin{array}{l}40 \text { weeks, } \\
4 \text { days }\end{array}$ & $\begin{array}{l}33 \text { weeks, } \\
6 \text { days }\end{array}$ \\
\hline Onset to delivery (days) & Post-partum 1 & Post-partum 1 & No & No & No & No & Post-partum 2 & Pre-partum 1 \\
\hline Fever on admission & No & No & No & No & No & No & No & Yes \\
\hline Post-partum fever & Yes & Yes & No & No & No & No & Yes & No \\
\hline Cough & No & No & No & No & No & No & No & No \\
\hline Other symptoms & No & No & No & No & No & No & No & No \\
\hline \multicolumn{9}{|l|}{ CT evidence of pneumonia } \\
\hline $\begin{array}{l}\text { Pre-partum typical signs of } \\
\text { viral infection }\end{array}$ & No & No & No & Yes & Yes & Yes & No & Yes \\
\hline $\begin{array}{l}\text { Post-partum typical signs of } \\
\text { viral infection }\end{array}$ & No & No & Yes & Yes & Yes & Yes & Yes & Yes \\
\hline SARS-CoV-2 RNA & Yes & Yes & Yes & Yes & Yes & Yes & NA & NA \\
\hline \multicolumn{9}{|l|}{ Delivery } \\
\hline Method of Delivery & C-section & C-section & C-section & C-section & $\begin{array}{l}\text { Vaginal } \\
\text { delivery }\end{array}$ & C-section & $\begin{array}{l}\text { Vaginal } \\
\text { delivery }\end{array}$ & C-section \\
\hline Indication for C-section & $\begin{array}{l}\text { History of } \\
\text { C-section }\end{array}$ & PROM & $\begin{array}{l}\text { Pre- } \\
\text { eclampsia }\end{array}$ & PROM & & $\begin{array}{l}\text { Fetal } \\
\text { distress }\end{array}$ & & $\begin{array}{l}\text { History of } \\
\text { C-section }\end{array}$ \\
\hline \multicolumn{9}{|l|}{ Treatment } \\
\hline Intensive unit care & No & No & No & No & No & No & No & No \\
\hline Mechanical ventilation & No & No & No & No & No & No & No & No \\
\hline
\end{tabular}

NA, not available; $C$-section; cesarean section; PROM, premature rupture of membrane.

Patient 1

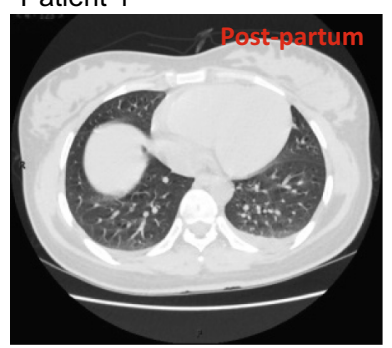

Patient 5

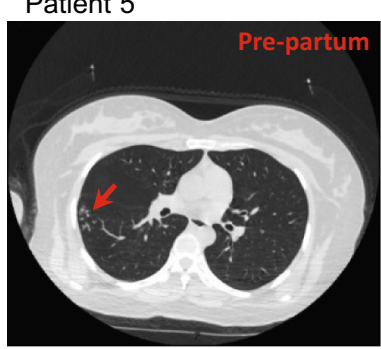

Patient 2

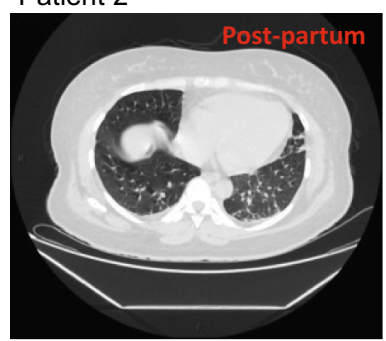

Patient 6

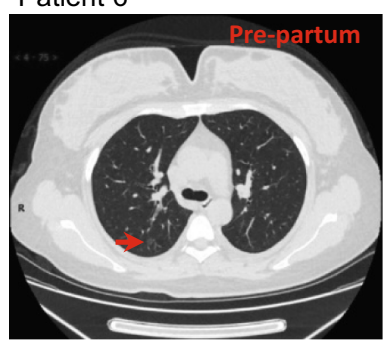

Patient 3

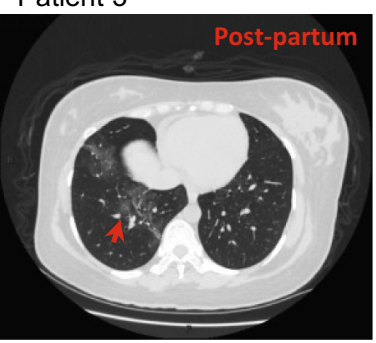

Patient 7

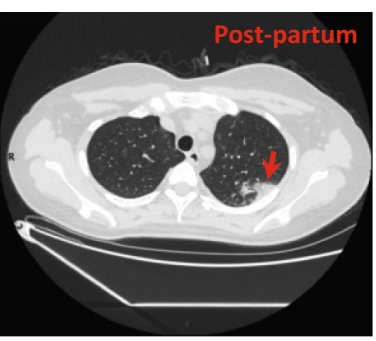

Patient 4

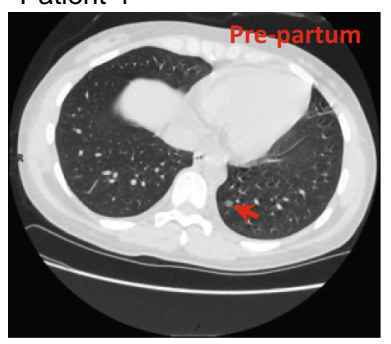

Patient 8

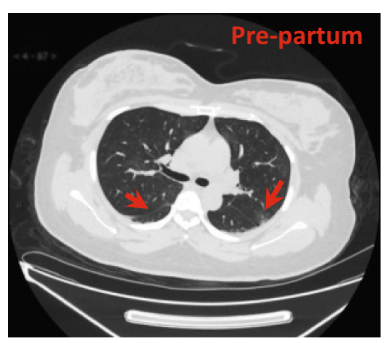

Fig. 1 Chest CT scans (transverse plane) of eight pregnant women with confirmed SARS-CoV-2 infection. Patients 1 and 2: no visible groundglass opacities or small plaque; Patient 3-8: ground-glass opacities or/and small plaque are indicated by red arrow.

The plasma D-dimer levels during the prepartum period were normal and comparable between pregnant women with SARS-CoV-2 infection and those without (Fig. 3B).
However, D-dimer levels of four patients with SARS-CoV-2 infection increased significantly postpartum, but did not change in the five patients without SARS-CoV-2 infection 


\section{A}

Pregnant women with SARS-CoV-2 Infection

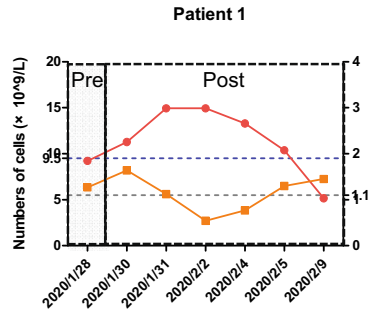

Patient 5

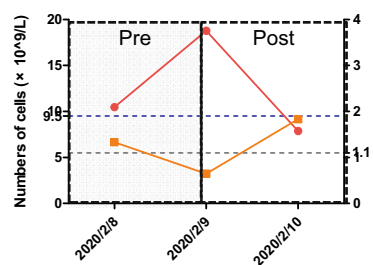

Patient 2

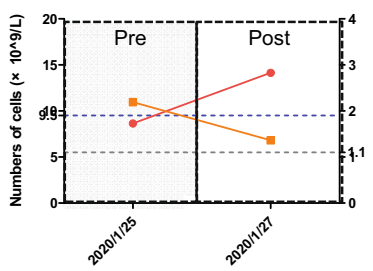

Patient 6

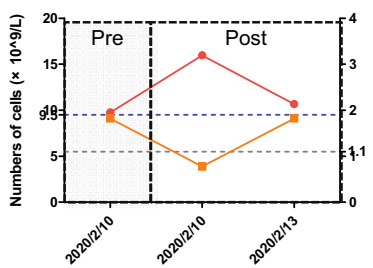

Patient 3

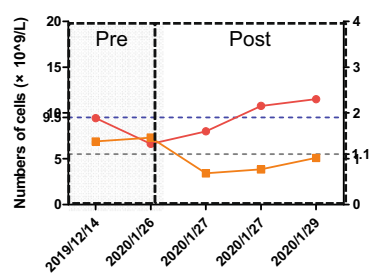

Patient 7

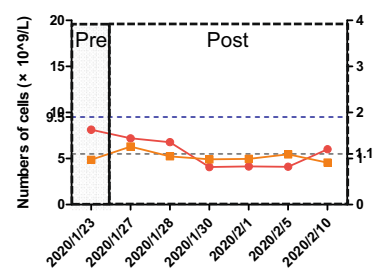

Patient 4

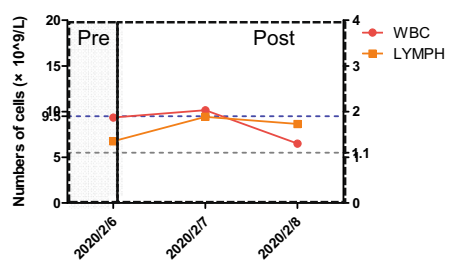

Patient 8

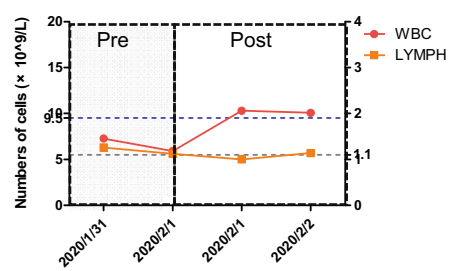

B

Pregnant women without SARS-CoV-2 Infection

Patient 1

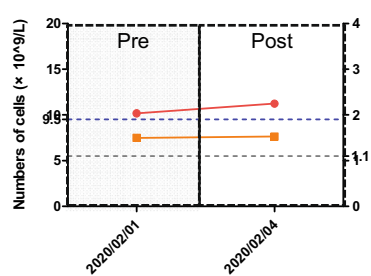

Patient 4

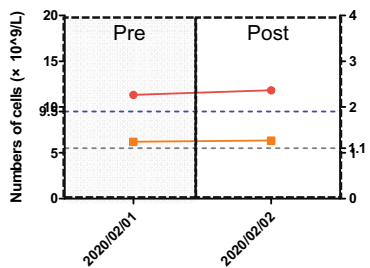

Fig. 2 Dynamic profile of white blood cells and lymphocyte count in pregnant women with or without severe acute respiratory syndrome coronavirus 2 (SARS-CoV-2) infection. Considering pregnant women with SARS-CoV-2 infection, patients $1-6$ are pregnant patients with laboratory-confirmed SARS-CoV-2 infection, and patients 7-8 are pregnant patients with highly suspected SARS-CoV-2 infection. The

(Fig. 3B and Supplementary Table S1). Both creatine kinase $(\mathrm{CK})$ and creatine kinase-MB (CK-MB) levels increased in four pregnant women with SARS-CoV-2 infection after delivery (Fig. 3C, 3D and Supplementary Table S1). In terms of other laboratory tests, all eight patients with confirmed or highly suspected SARS-CoV-2 infection had normal levels of liver enzymes.

All patients received empirical antibiotic treatment and supportive care. None of the eight patients required intensive care or mechanical ventilation. left $y$-axis shows the number of white blood cells (WBC). The right $y$ axis shows the number of lymphocytes (LYMPH). The dotted lines in blue show the upper normal limit of the WBC count, and the dotted line in grey shows the lower normal limit of LYMPH count. Pre, prepartum; Post, post-partum.

\section{Discussion}

This study reported new SARS-CoV-2 infected pregnant patients and revealed distant clinical presentations during the pre- and post-partum periods. Comprehensive data from clinical manifestations, laboratory examinations, and chest imaging were subsequently analyzed.

During pregnancy, several mechanical and pathophysiological adaptive changes occur in the respiratory system, including a decrease in respiratory volumes, increased oxygen consumption, and edema of the respiratory tract mucosa, which can render them intolerant to hypoxia. 
Fig. 3 The levels of inflammatory factors and kinase in pregnant women at prepartum and post-partum. The pregnant women with SARSCoV-2 infection included six pregnant patients with laboratory-confirmed SARSCoV-2 infection and two pregnant patients with highly suspected SARS-CoV-2 infection. The participants without the SARS-CoV-2 infection. The levels of C-reactive protein (CRP), D-dimer, creatine kinase $(\mathrm{CK})$, and creatine kinase-MB (CK-MB) in the serum or plasma were detected.
A
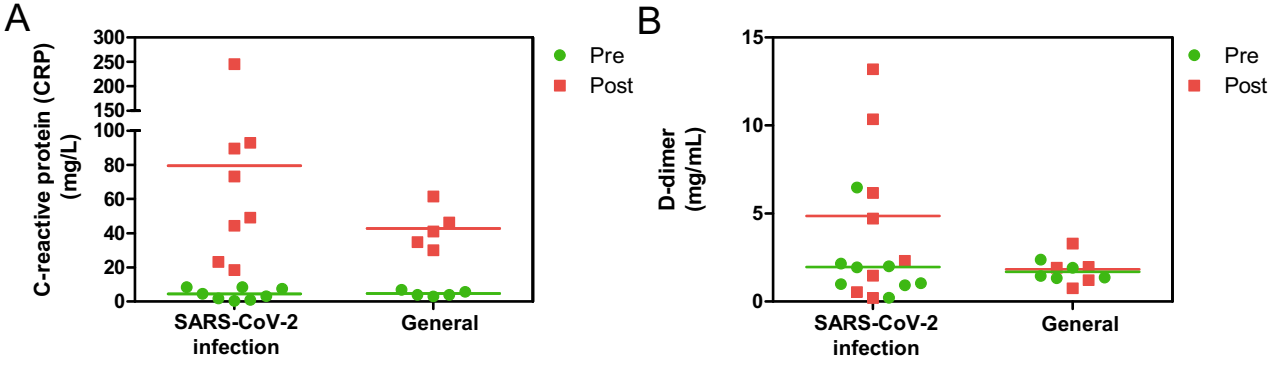

C

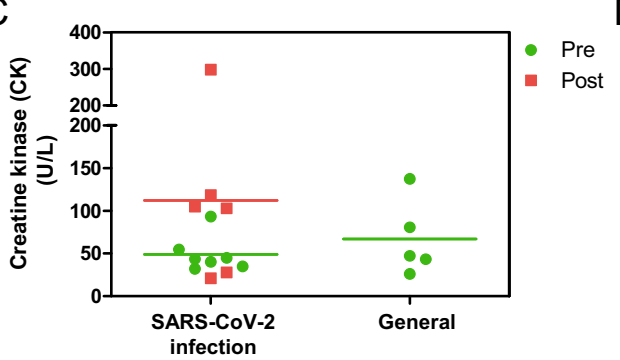

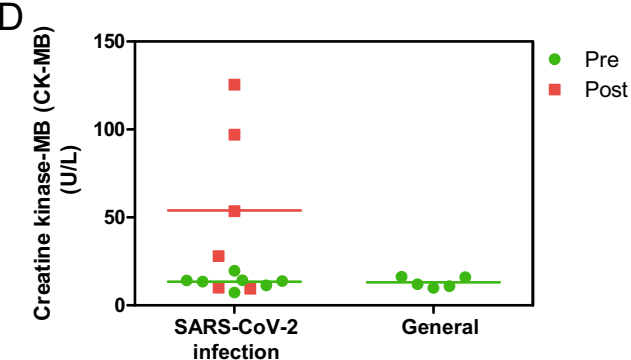

Moreover, immune adaptations are required to accommodate the fetus (Kourtis et al. 2014). Therefore, pregnant women are particularly susceptible to respiratory pathogens and severe pneumonia. Previous studies have reported relatively more severe cases with higher mortality rates among pregnant women upon contracting respiratory viruses such as influenza virus and SARS virus (Gottfredsson 2008; Jamieson et al. 2009; Wong et al. 2004). Pregnant SARS-CoV-2 patients reportedly presented with a similar pattern of clinical characteristics to that of nonpregnant adult patients (Chen $\mathrm{H}$ et al. 2020; Huang et al. 2020). An investigation in 138 hospitalized patients with COVID showed that $98.6 \%$ patients had clinical manifestations of fever, $69.6 \%$ patients had fatigue, and $59.4 \%$ patients had dry cough. In addition, $70.3 \%$ patients had lymphopenia and $39.9 \%$ had elevated lactate dehydrogenase. Chest computed tomographic (CT) scans showed that all patients presented bilateral patchy shadows or ground glass opacity in the lungs of all patients (Wang et al. 2020). Another descriptive study in 99 cases with COVID reported that $83 \%$ patients had fever, $82 \%$ patients had cough, and $31 \%$ patients had shortness of breath. Other clinical manifestations include muscle ache, confusion, headache, sore throat and so on. The imaging examination showed that $75 \%$ patients presented bilateral pneumonia, $14 \%$ patients showed multiple mottling and ground-glass opacity (Chen N et al. 2020). Here, four patients had no symptoms during the observation period; additionally, the only symptom observed in the remaining patients was fever. The liver enzyme levels in all eight patients were also normal. As far as the imaging features were concerned, the typical signs of viral infection-ground-glass opacity in the lungs were also mild. Therefore, according to our study, SARS$\mathrm{CoV}-2$ infected pregnant patients appeared to present with relatively milder clinical symptoms than non-pregnant adult patients.

Our study revealed that four of the eight pregnant women were asymptomatic before delivery but became symptomatic after delivery. Correspondingly, WBC counts increased while LYMPH counts decreased in most pregnant patients with SARS-CoV-2 infection. Furthermore, CRP levels increased considerably higher than those observed during general pregnancy. During pregnancy, the concentrations of steroid hormones such as estrogens and progesterone progressively increase over the course of pregnancy (Vojtek et al. 2018). In addition, to ensure that the mother's body can actively tolerate the semi-allogeneic fetus, the immune system is modulated (Vojtek et al. 2018). However, there is a sudden and substantial decrease in steroid hormone concentrations during the immediate postpartum period (Dennis et al. 2008). In comparison to the third trimester of pregnancy, there is a significant change in immune cells including the blood phagocytes, pDCs, NK cells, and T cells during the post-partum period (Kraus et al. 2012). It is possible that these alterations in both the hormones and immune system trigger the onset of SARS-CoV-2 infection following partum. Further investigations are needed to elucidate the immunological differences between pre- and post-partum in the context of combating a SARS-CoV-2 infection.

Summarily, our results suggest that compared to nonpregnant adult patients, pneumonia and other symptoms were mild in pregnant patients with SARS-CoV-2 infection. A number of pregnant women presented with asymptomatic SARS-CoV-2 infection pre-partum, but the disease onset may occur post-partum. Close monitoring of laboratory parameters such as WBC count, LYMPH count, and CRP as well as the imaging features in chest CT scans, 
may be helpful for the early prevention, diagnosis, and treatment of SARS-CoV-2 infection during pregnancy.

Acknowledgements We thank Bo Nie, Zuliang Shi and Dan Lu in Department of Laboratory Medicine, Maternal and Child Health Hospital of Hubei Province for kindly helping us to collect all the data. We thank Dr. Quan Gan in Department of Intensive Care Unit, Maternal and Child Health Hospital of Hubei Province for kindly helping us to discuss clinical data.

Author Contributions CW, WR and JX conceptualized and designed the study, drafted the initial manuscript, and reviewed and revised the manuscript. WY, XW, TZ and YZ collected data and carried out the initial analyses.

\section{Compliance with Ethical Standards}

Conflict of interest The authors declare that they have no conflict of interest.

Animal and Human Rights Statement This study was approved by the Medical Ethical Committee of the Maternal and Child Health Hospital of Hubei Province. Written informed consent was obtained from the spouses of each enrolled patient.

\section{References}

Chen N, Zhou M, Dong X, Qu J, Gong F, Han Y, Qiu Y, Wang J, Liu Y, Wei Y, Xia J, Yu T, Zhang X, Zhang L (2020) Epidemiological and clinical characteristics of 99 cases of 2019 novel coronavirus pneumonia in Wuhan, China: a descriptive study. Lancet 395:507-513

Chen H, Guo J, Wang C, Luo F, Yu X, Zhang W, Li J, Zhao D, Xu D, Gong Q, Liao J, Yang H, Hou W, Zhang Y (2020) Clinical characteristics and intrauterine vertical transmission potential of COVID-19 infection in nine pregnant women: a retrospective review of medical records. Lancet 395:809-815

Dennis CL, Ross LE, Herxheimer A (2008) Oestrogens and progestins for preventing and treating postpartum depression. Cochrane Database Syst Rev. https://doi.org/10.1002/14651858. CD001690.pub2

Gottfredsson M (2008) The Spanish flu in Iceland 1918. Lessons in medicine and history. Laeknabladid 94:737-745 (in Icelandic)

Huang C, Wang Y, Li X, Ren L, Zhao J, Hu Y, Zhang L, Fan G, Xu J, Gu X, Cheng Z, Yu T, Xia J, Wei Y, Wu W, Xie X, Yin W, Li H, Liu M, Xiao Y, Gao H, Guo L, Xie J, Wang G, Jiang R, Gao Z, Jin Q, Wang J, Cao B (2020) Clinical features of patients infected with 2019 novel coronavirus in Wuhan, China. Lancet 395:497-506

Hui DS, Azhar EI, Madani TA, Ntoumi F, Kock R, Dar O, Ippolito G, Mchugh TD, Memish ZA, Drosten C, Zumla A, Petersen E
(2020) The continuing 2019-nCoV epidemic threat of novel coronaviruses to global health-The latest 2019 novel coronavirus outbreak in Wuhan, China. Int J Infect Dis 91:264-266

Jamieson DJ, Honein MA, Rasmussen SA, Williams JL, Swerdlow DL, Biggerstaff MS, Lindstrom S, Louie JK, Christ CM, Bohm SR, Fonseca VP, Ritger KA, Kuhles DJ, Eggers P, Bruce H, Davidson HA, Lutterloh E, Harris ML, Burke C, Cocoros N, Finelli L, MacFarlane KF, Shu B, Olsen SJ, Novel Influenza A (H1N1) Pregnancy Working Group (2009) H1N1 2009 influenza virus infection during pregnancy in the USA. Lancet 374:451-458

Kourtis AP, Read JS, Jamieson DJ (2014) Pregnancy and infection. N Engl J Med 370:2211-2218

Kraus TA, Engel SM, Sperling RS, Kellerman L, Lo Y, Wallenstein S, Escribese MM, Garrido JL, Singh T, Loubeau M, Moran TM (2012) Characterizing the pregnancy immune phenotype: results of the viral immunity and pregnancy (VIP) study. J Clin Immunol 32:300-311

Lu H, Stratton CW, Tang YW (2020) Outbreak of pneumonia of unknown etiology in Wuhan, China: the mystery and the miracle. J Med Virol 92:401-402

National Health Commission of the PRC (2020) New coronavirus pneumonia prevention and control program (trial version 7). http://www.nhc.gov.cn/yzygj/s7653p/202003/46c9294a7dfe4 cef80dc7f5912eb1989.shtml. Accessed 4 Mar 2020

Robinson DP, Klein SL (2012) Pregnancy and pregnancy-associated hormones alter immune responses and disease pathogenesis. Horm Behav 62:263-271

Sappenfield E, Jamieson DJ, Kourtis AP (2013) Pregnancy and susceptibility to infectious diseases. Infect Dis Obstet Gynecol 2013:752852

Subramaniam S, Scharrer I (2018) Procoagulant activity during viral infections. Front Biosci (Landmark Ed) 23:1060-1081

Vojtek I, Dieussaert I, Doherty TM, Franck V, Hanssens L, Miller J, Bekkat-Berkani R, Kandeil W, Prado-Cohrs D, Vyse A (2018) Maternal immunization: where are we now and how to move forward? Ann Med 50:193-208

Wang D, Hu B, Hu C, Zhu F, Liu X, Zhang J, Wang B, Xiang H, Cheng Z, Xiong Y, Zhao Y, Li Y, Wang X, Peng Z (2020) Clinical characteristics of 138 hospitalized patients with 2019 novel coronavirus-infected pneumonia in Wuhan, China. JAMA. https://doi.org/10.1001/jama.2020.1585

Wong SF, Chow KM, Leung TN, Ng WF, Ng TK, Shek CC, Ng PC, Lam PW, Ho LC, To WW, Lai ST, Yan WW, Tan PY (2004) Pregnancy and perinatal outcomes of women with severe acute respiratory syndrome. Am J Obstet Gynecol 191:292-297

World Healthy Organization (WHO) (2020) Clinical management of severe acute respiratory infection when novel coronavirus (nCoV) infection is suspected. https://www.who.int/publica tions-detail/clinical-management-of-severe-acute-respiratoryinfection-when-novel-coronavirus-(ncov)-infection-is-suspected. Accessed 13 Mar 2020

Wu Y, Potempa LA, El Kebir D, Filep JG (2015) C-reactive protein and inflammation: conformational changes affect function. Biol Chem 396:1181-1197 